\title{
Case Report Syncope Caused by Huge Hiatal Hernia
}

\author{
Gabriel Vanerio \\ Intensive Care Unit and Cardiology Departments, British Hospital Montevideo, 2420 Avenue Italia, 11600 Montevideo, Uruguay \\ Correspondence should be addressed to Gabriel Vanerio, gvanerio@yahoo.com
}

Received 19 June 2011; Accepted 18 July 2011

Academic Editors: R. Akdemir and T. Tak

Copyright ( $) 2011$ Gabriel Vanerio. This is an open access article distributed under the Creative Commons Attribution License, which permits unrestricted use, distribution, and reproduction in any medium, provided the original work is properly cited.

\begin{abstract}
A 84-year-old white female had a brief loss of consciousness while playing bridge. A few minutes before the episode she had eaten pizza and significant amount of carbonated soft drinks. After recovery, her friends noticed that she was alert, but pale and sweating. Upon arrival at the emergency room, sitting blood pressure was $160 / 60 \mathrm{mmHg}$ with a normal sinus rhythm. A chest XRay was performed, which was essential to make the diagnosis. The X-Ray showed a large retrocardiac opacity with air and liquid level compatible with a giant hiatus hernia. After a copious snack the hiatal hernia compressed the left atrium, decreasing the left cardiac output, elucidating the mechanism of the syncopal episode. In patients presenting with swallow syncope (particularly after a copious meal, validating the importance of a careful history), a chest X-Ray should be always be performed.
\end{abstract}

Patients presenting to the emergency department with syncope are occasionally a diagnostic challenge. The cause of syncope might be revealed by a careful history and physical examination in approximately $40 \%-60 \%$ of patients.

The chest X-Ray is a common test in patients presenting with syncope, but its value is less well-established. Unless guided by the history and physical examination findings, it is unlikely that a routine chest X-Ray will uncover the cause of syncope. We present a patient where the chest X-Ray illustrated the cause of syncope.

A 84-year-old white female with previous history of hypothyroidism and arterial hypertension had a brief loss of consciousness, while she was playing bridge. A few minutes before the episode she had consumed a significant amount of pizza and a carbonated soft drink. After recovery, her friends noticed that she was alert but pale and sweating. Upon arrival at the emergency room, she had a blood pressure of $160 / 60 \mathrm{mmHg}$ with a normal sinus rhythm. The chest XRay (AP and lateral view) is shown below Figure 1(a). A large retrocardiac opacity is observed with air and liquid level compatible with a giant hiatus hernia. A CT-Scan with reconstruction, shown in the lower Figure 1(b), established the diagnosis. The mass is located behind the heart and in close relation with the left atrium.

The cause of the syncopal episode might be related to the left atrial compression due to the sudden enlargement of the stomach. The patient underwent repair-reconstructive surgery (Nissen's fundoplication) with excellent result and no more syncopal episodes.

Syncope is induced by various conditions. Swallow syncope, a vagally mediated reflex, constitutes a rare cause of syncope [1-6].

Some foods or beverages such as cold water, hot liquid, or carbonated drinks have been reported to trigger syncopal attacks.

Despite swallow syncope caused by compression of the left atrium due to a huge hiatal hernia has been described previously [1-5], the chest X-Ray is the appropriate diagnostic test in this unusual type of syncope.

We emphasize that a patient presenting to the emergency room with swallow syncope (particularly after an important amount of liquids), a chest X-Ray (AP and lateral views) should always be performed.

In conclusion, we present a case of postprandial syncope in a patient with a large hiatal hernia that probably compressed the left atrium, thus causing an obstructive cardiac lesion with resultant syncope.

\section{Conflict of Interests}

The authors declares that there is no conflict of interests. 

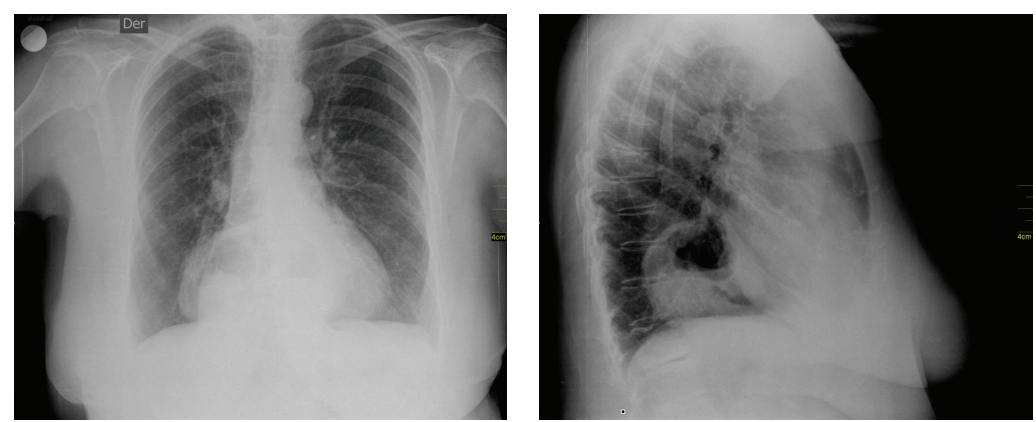

(a)
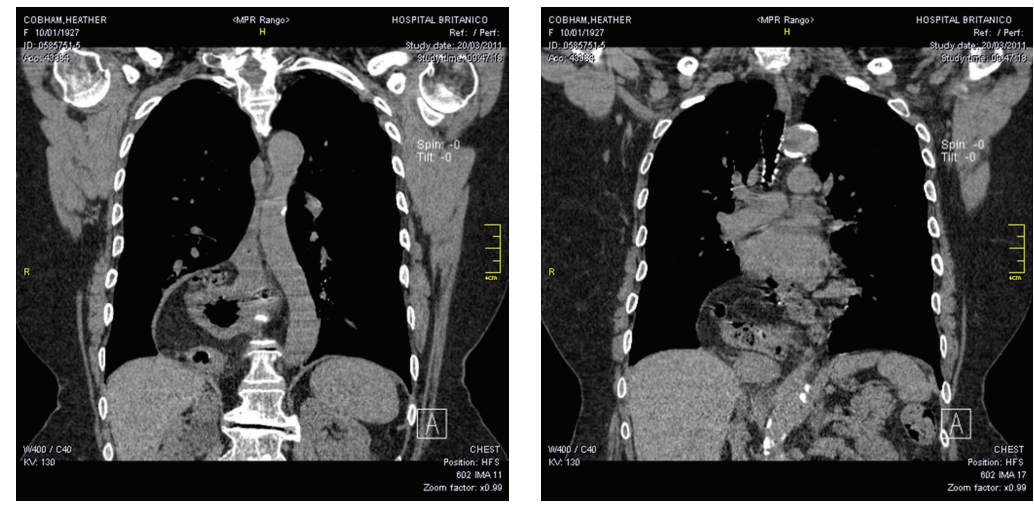

(b)

Figure 1: (a) A large retrocardiac opacity is observed with air and liquid level compatible with a giant hiatus hernia. (b) A CT-scan with reconstruction is shown validating the diagnosis. The mass corresponding to the stomach and likely small bowel is located behind the heart and in close relation to the left atrium.

\section{References}

[1] E. Favaretto, N. Schenal, N. Russo, G. Buja, S. Iliceto, and C. Bilato, "An uncommon case of right-sided throat pain and swallow syncope," Journal of Cardiovascular Medicine, vol. 9, no. 11, pp. 1152-1155, 2008.

[2] C. Hausmann Hartsleben, "A 57-year-old patient with swallow syncope while swallowing," Praxis, vol. 93, no. 17, pp. 725-727, 2004.

[3] Y. Oishi, T. Ishimoto, N. Nagase et al., "Syncope upon swallowing caused by an esophageal hiatal hernia compressing the left atrium: a case report," Echocardiography, vol. 21, no. 1, pp. 61-64, 2004.

[4] T. Maekawa, M. Suematsu, T. Shimada, G. O. Masayoshi, and T. Shimada, "Unusual swallow syncope caused by huge hiatal hernia," Internal Medicine, vol. 41, no. 3, pp. 199-201, 2002.

[5] I. Akdemir, V. Davutoglu, and S. Aktaran, "Giant hiatal hernia presenting with stable angina pectoris and syncope: a case report," Angiology, vol. 52, no. 12, pp. 863-865, 2001.

[6] T. D. Karamitsos, J. R. Arnold, O. J. Rider et al., "Massive hiatus hernia impeding transoesophageal echocardiography in a patient with swallow-syncope syndrome," Hellenic Journal of Cardiology, vol. 50, no. 3, pp. 216-217, 2009. 


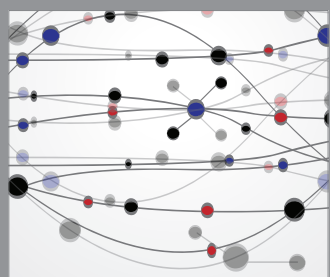

The Scientific World Journal
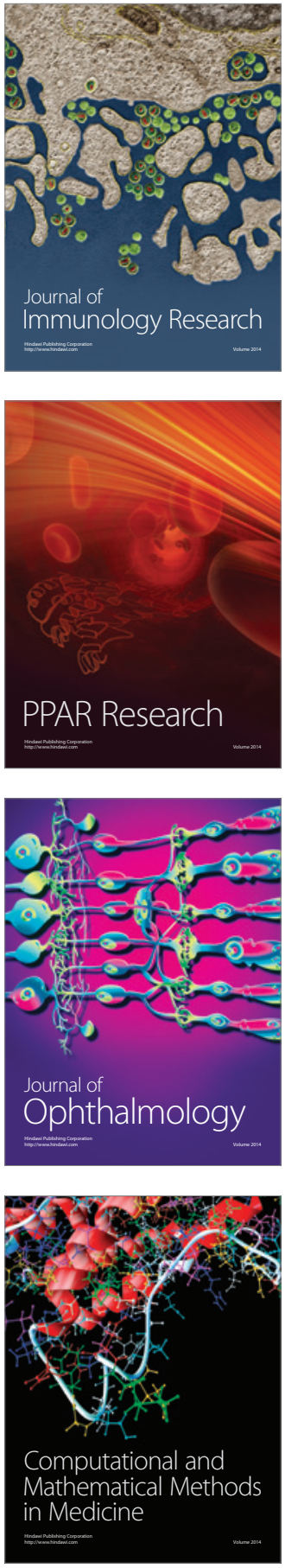

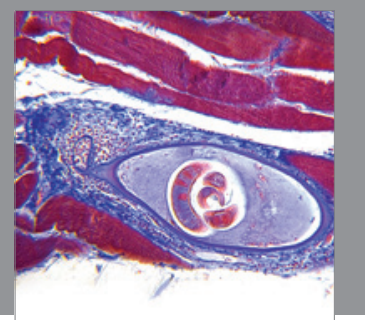

Gastroenterology

Research and Practice
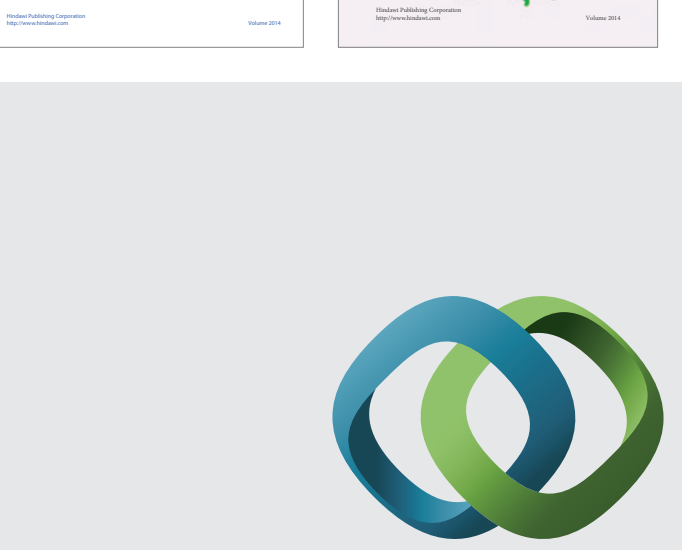

\section{Hindawi}

Submit your manuscripts at

http://www.hindawi.com
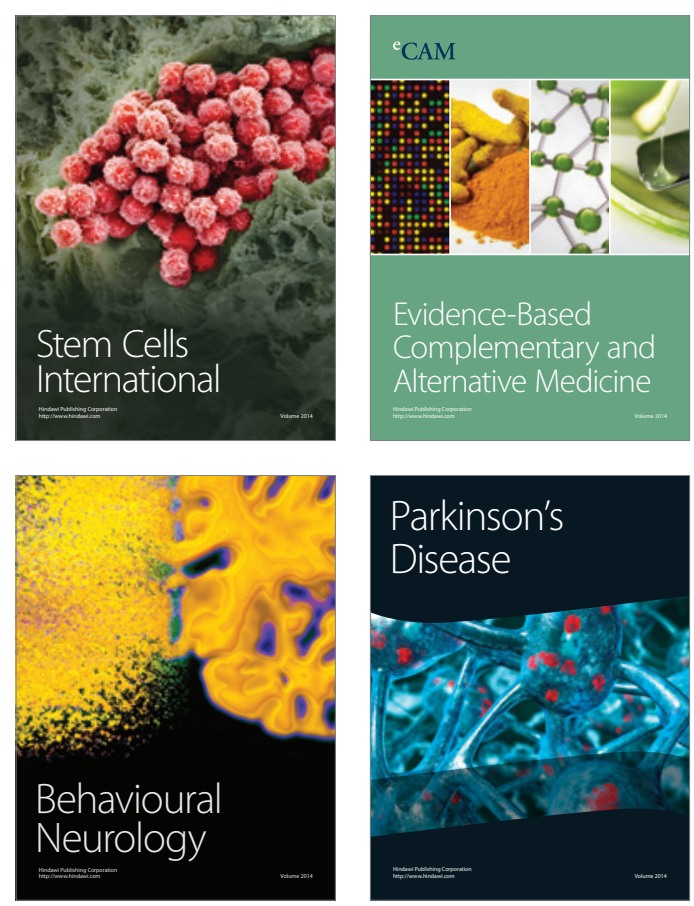

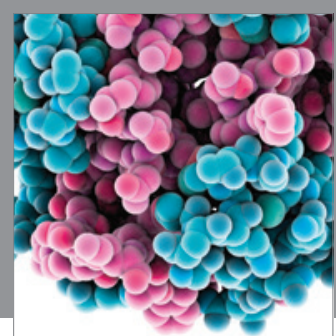

Journal of
Diabetes Research

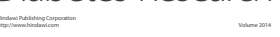

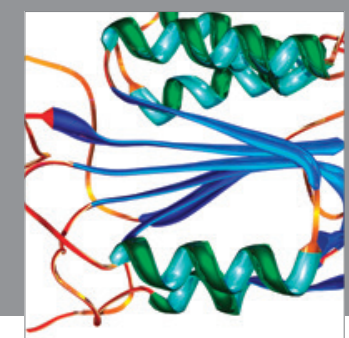

Disease Markers
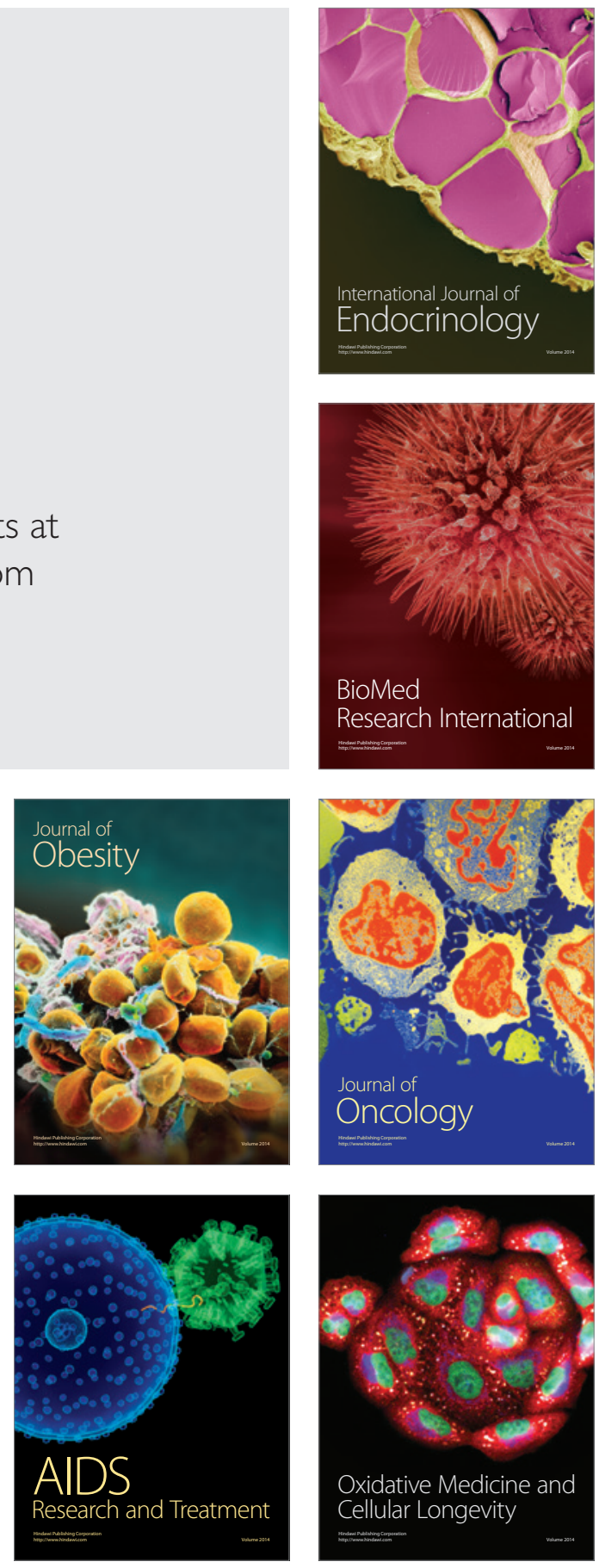